\section{In the dock}

EVERY desert scene comes with scorpions, but these rugged animals are found in various habitats. T. G. Benton has been studying the thriving population of Euscorpius flavicaudis which has been resident in the dockyards of Sheerness in southern England, possibly since victorian times (J. Zool., Lond. 226, 351-368; 1992). Benton made 10,000 sightings of the creatures, and estimates that the population is close to 700 . But the animals are highly reclusive, spending almost all their time hidden in cracks in the dock walls (females, for instance, leave their holes on average fewer than ten times a year). Otherwise they behave very much as if they lived in the desert. Scorpion lifestyle is hardly influenced by abiotic factors such as temperature: this, suggests Benton, is the key to their adaptability.

\section{Where the ions are}

PROTEINS are polyions, bristling with charged groups at their surfaces, the electroneutrality of which is maintained by counterions. To what extent these occupy unique structural sites, rather than being merely more concentrated within the protein domain, is a question that has agitated chemists since Donnan. Crystallography gives the means of resolving the riddle and 0 . Gursky et al. (Biophys. J. 64, 604-611; 1992) have tackled it by reference to one of the smallest of globular proteins, insulin. Sodium ions cannot be distinguished by scattering from water and Gursky et al. replaced them by other univalent cations, in particular thallium. Two site-bound cations were found, one in an enclosed cavity, the other trapped between two insulin molecules, and each interacting apparently with a pair of carbonyl dipoles. The greater part of the neutralization comes from counterions at large in the solution.

\section{Light spin}

Physicists are becoming adept at manipulating atoms and molecules in beams of laser radiation. Optical molasses cool atoms through viscous friction; and optical tweezers trap atoms with light pressure. Another concept of fluid mechanics creeps into these studies with the report from $A$. Hemmerich and T. W. Hänsch of optical vortices (Phys. Rev. Lett. 68, 1492$1495 ; 1992)$. The authors fired a beam of rubidium atoms through the intersection of a pair of crossed laser beams. With the beams in phase, the atoms emerged unscathed, the radiation pressure being isotropic. With the lasers out of phase, off-axis pressure vortices pulled the atom beam's edges visibly out of shape. The authors expect that optical molasses, with three lasers and special polarization conditions, could give highly unusual effects. lating structure with properties.

The fact that $a_{0}$ rigorously controls $T_{\mathrm{c}}$ was easily explained (once observed) by nearly every model of superconductivity. Any theory of superconductivity starts with some mechanism, exotic or prosaic, to create a condensate of paired electrons, which can carry a supercurrent through the lattice. Independent of the precise specification of that mechanism, the rules of weak-coupling 'BCS' theory of superconductivity hold that the transition temperature rises sharply as the number of electrons able to carry the supercurrent (those at the 'Fermi' energy) increases. As the lattice expands, there is less quantum-mechanical overlap and less interaction between electrons on adjacent fullerenes and the energy spanned by the electronic band becomes narrower; consequently more electrons find themselves near the Fermi energy. (Unfortunately, it is not obvious that $T_{\mathrm{c}}$ could continue to rise for bandwidths less than the electron pairing energy.) So the fact that $T_{\mathrm{c}}$ is inversely related to $a_{0}$ does not reveal anything directly about the particular mechanism of superconductivity. Other experiments, such as the variation of $T_{c}$ with isotopic substitution, may do so, but the dust has not yet entirely settled.

This simple state of affairs has been subverted by the discoveries reported in this issue. Workers at AT\&T Bell Laboratories, who report on page $416^{1}$, have doped fullerene with the smaller alkali, $\mathrm{Na}$, and found no sign of superconductivity. This material has a value of $a_{0}$ which would have led one to expect superconductivity at $T_{\mathrm{c}}$ around $16 \mathrm{~K}$; we have clearly fallen off the established curve of $T_{\mathrm{c}}$ against $a_{0}$. The authors find a reason for this disruption, in that the $\mathrm{Na}_{3} \mathrm{C}_{60}$ apparently disproportionates into $\mathrm{Na}_{2} \mathrm{C}_{60}$ and $\mathrm{Na}_{6} \mathrm{C}_{60}$ as it is cooled. Indeed, recent photoelectron-spectroscopic measurements partially anticipated this result by showing that both $\mathrm{Na}$ - and Li-doped fullerides are insulators across the entire range of compositions ${ }^{6}$.

In a related article, on page $419^{2}$, a group from NEC Fundamental Research Laboratories, Japan, explores material doped with mixtures of $\mathrm{Na}, \mathrm{Li}$ and heavier alkalis. The researchers find that these materials do superconduct, but with $T_{\mathrm{c}}$ values far below what is expected from their lattice constants. For example, $\mathrm{Na}_{2} \mathrm{RbC}_{60}$ has a $T_{\mathrm{c}}$ of $2.5 \mathrm{~K}$, whereas $\mathrm{K}_{3} \mathrm{C}_{60}$ compressed to the same

$a_{0}$ of $14.028 \AA$ becomes superconducting at $10.5 \mathrm{~K}$. Both papers show that the inclusion of smaller alkali ions actually compresses $a_{0}$ to below that of the undoped $\mathrm{C}_{60}$. It appears that such an internal compression is strongly inimical to superconductivity.

The time also seems right for stuffing an unexpected excess of cations into fullerene crystal lattices. Previously known cubic-close-packed fullerides have the stoichiometry $\mathrm{A}_{3} \mathrm{C}_{60}$, with one alkali ion in each interstitial space of the

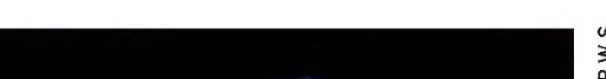

